\title{
Appropriate terms for chest CT features in COVID-19 infection
}

\author{
Michele Scialpi ${ }^{1} \cdot$ Irene Piscioli $^{2} \cdot$ Antonio Improta $^{1} \cdot$ Danilo Delli Carpini $^{1} \cdot$ Francesco Mancioli ${ }^{3}$
}

Received: 27 May 2020 / Accepted: 3 June 2020 / Published online: 15 June 2020

(C) Japan Radiological Society 2020

Dear editor,

We have read with interest the article by Dr. Iwasawa and colleagues [1] published in the Japanese Journal of Radiology issue of May 2020. The authors reported on ultra-highresolution CT (U-HRCT) the hallmarks of COVID-19 pneumonia, and described for the first time the alveolar collapse.

Some points are needed to be discussed and clarified in regards to pneumonia and alveolar collapse in COVID-19.

Similarly to others, the authors attributed chest CT parenchymal features (ground-glass opacity, consolidation, linear opacity, crazy paving) to COVID-19 pneumonia [1].

Direct endothelial damage determined by COVID-19 [2] and a detailed autopsy series of predominantly Caucasian COVID-19 patients [3] demonstrated that pneumonia is not the only histopathological lesion in the lung tissue. In COVID-19, the respiratory failure represents the primary cause of death: in the lung tissue of patients who died, the pattern of pneumonia ranges from 0 to $48 \%$ of cases $[2,3]$. Other pathological features in the lung tissues of patients who died with COVID-19 such as exudative diffuse alveolar damage with massive capillary congestion, microthrombi, moderate intraalveolar fibrin exudation, pulmonary embolism, alveolar hemorrhage and vasculitis may determine similar patterns to those of pneumonia on chest CT in COVID-19 patients. Therefore, some discrepancy with respect to clinical diagnoses has been demonstrated, since cases clinically diagnosed as "pneumonia, COVID-related" only showed signs of diffused alveolar damage and no signs of suppurative bronchopneumonia both macroscopically and histologically [3].

Michele Scialpi

michelescialpi1@gmail.com

1 Division of Diagnostic Imaging, Department of Surgical and Biomedical Sciences, Santa Maria della Misericordia Hospital, University of Perugia, S. Andrea Delle Fratte, 06156 Perugia, Italy

2 Division of Radiology, Budrio Hospital, Bologna, Italy

3 Division of Radiology, Azienda Ospedaliera Santa Maria, Terni, Italy
By using U-HRCT, Dr Iwasawa and colleagues described for the first time the alveolar collapse resulting in a local lung volume loss. In the histopathologic literature, the alveolar collapse is synonym of atelectasis, a secondary phenomenon causing loss of pulmonary tissue with collapse of numerous alveolar cavities. So, the term "alveolar collapse" should be replaced with "atelectasis" whose histological and radiological features are well known. In the autopsy study by Menter et al. [3] no case of atelectasis was found.

In conclusion, an appropriate terminology is necessary for the radiological diagnosis and the pulmonary CT findings should be confirmed by autopsy studies. In severe COVID19 patients, based on the pathophysiology and histological data the pulmonary alterations are not exclusively attributable to pneumonia [4]; the term alveolar collapse is improper and should be replaced by atelectasis.

\section{References}

1. Iwasawa T, Sato M, Yamaya T, et al. Ultra-high-resolution computed tomography can demonstrate alveolar collapse in novel coronavirus (COVID-19) pneumonia. Jpn J Radiol. 2020;38:394-8.

2. Ackermann M, Verleden SE, Kuehnel M, et al. Pulmonary vascular endothelialitis, thrombosis, and angiogenesis in Covid-19. Eng J Med. 2020;21:20-122. https://doi.org/10.1056/NEJMoa2015 432.

3. Menter T, Haslbauer JD, Nienhold R, et al. Post-mortem examination of COVID19 patients reveals diffuse alveolar damage with severe capillary congestion and variegated findings of lungs and other organs suggesting vascular dysfunction. Histopathology. 2020;4:12.

4. Scialpi M, Scialpi S, Irene P, et al. Pulmonary thromboembolism in critical ill COVID-19 patients. Int J Infect Dis. 2020;95:361-2. https://doi.org/10.1016/j.ijid.2020.04.056.

Publisher's Note Springer Nature remains neutral with regard to jurisdictional claims in published maps and institutional affiliations. 